\title{
Measuring inspiratory muscle strength in neuromuscular disease: one test or two?
}

\author{
N. Terzi*, D. Orlikowski*, C. Fermanian*, M. Lejaille*, L. Falaize*, A. Louis*, \\ J-C. Raphael*, B. Fauroux" and F. Lofaso*,
}

ABSTRACT: Inspiratory muscle strength monitoring is crucial in patients with neuromuscular disorders. The sniff nasal inspiratory pressure (SNIP) and maximal inspiratory pressure ( $\left.P_{I, \max }\right)$ are usually measured. The present study investigated whether the test yielding the best value at baseline continued to yield the best value during follow-up.

The present study included 25 patients with Duchenne muscular dystrophy (DMD) and 61 with myotonic muscular dystrophy (MMD). SNIP and PI,max were measured at baseline and then annually.

At baseline, SNIP was lower than PI,max in 20 (80\%) DMD patients and 32 (52\%) MMD patients. During follow-up in DMD patients, changes in the best method always occurred from SNIP to PI,max. In MMD patients, when SNIP was better than PI,max at baseline, SNIP was usually (88\%) better during follow-up, whereas a better PI,max than SNIP at baseline was frequently (50\%) followed by a shift to SNIP.

Maximal inspiratory pressure may be sufficient for monitoring inspiratory muscle function in Duchenne muscular dystrophy adults. In myotonic muscular dystrophy, the marked variability in the test yielding the best value at baseline indicates a need for performance of both tests at baseline. However, when sniff nasal inspiratory pressure measurement yields the best value at baseline, using sniff nasal inspiratory pressure alone during follow-up may be appropriate.

KEYWORDS: Maximal inspiratory pressure, neuromuscular disease, sniff pressure

$\mathbf{R}$ espiratory pump failure is one of the main causes of death in patients with neuromuscular disorders. Therefore, respiratory muscle strength must be monitored closely in order to evaluate the risk of respiratory failure. The static maximal mouth pressure measured during a maximal inspiratory effort that is sustained for $\geqslant 1 \mathrm{~s}$ against an occluded airway is the most widely used parameter for assessing inspiratory muscle strength [1]. However, this static maximal inspiratory pressure $(P \mathrm{I}, \max )$ manoeuvre is difficult to perform, and the extent to which low values reflect inspiratory muscle weakness and/or poor motivation or coordination of the patient cannot be determined. Moreover, several independent variables, such as technical factors (most notably the type of mouthpiece used) and the time allowed for learning the manoeuvre, may lead to variations across laboratories [2, 3]. Sniffing is a natural effort that many patients find easier to perform than static efforts, and sniff nasal inspiratory pressure (SNIP) is a recently suggested alternative to $P \mathrm{I}, \max [4,5]$. In patients with neuromuscular disorders, each of these two volitional tests has its own advantages and disadvantages, such that the two tests are complementary rather than interchangeable [6-8].

Since patients require distinctive skills and features to perform well on both of these volitional techniques [4], it was hypothesised that the test yielding the highest value at baseline for each patient continues to yield the highest value during follow-up and, therefore, could be used alone during follow-up. This hypothesis was evaluated in two of the most common chronic neuromuscular diseases: Duchenne muscular dystrophy (DMD), a disease confined to the skeletal muscle; and Steinert myotonic muscular dystrophy (MMD), which is characterised by a slower progression of muscle weakness than in DMD patients and frequent central nervous system involvement, responsible for cognitive impairment.

\section{METHODS}

\section{Patients}

The present study was approved by the appropriate ethics committee (Ambroise Paré Teaching Hospital, Paris, France) and informed consent was obtained from all study participants. Patients

\section{AFFILIATIONS}

*Intensive Care Unit, Dept of

Physiology-Functional Testing and Centre for Technological Innovation, Raymond Poincaré Teaching Hospital, Garches,

\#Paediatric Pulmonary Dept and National Institute for Health and Medical Research (INSERM) Mixed Research Unit 719, Armand Trousseau Teaching Hospital, Pierre and Marie Curie University, Paris, and,

"INSERM Mixed Research Unit 841 , Créteil, France.

CORRESPONDENCE

F. Lofaso

Service de Physiologie-Explorations Fonctionnelles

Hôpital Raymond Poincaré

92380 Garches

France

Fax: 33147107943

E-mail: f.lofaso@rpc.ap-hop-paris.fr

Received:

July 252007

Accepted after revision:

September 072007

STATEMENT OF INTEREST

None declared.

European Respiratory Journal Print ISSN 0903-1936 Online ISSN 1399-3003 
were recruited between January 2002 and December 2005 during routine follow-up visits at the Raymond Poincaré Teaching Hospital (Garches, France). Consecutive patients who had a diagnosis of DMD or MMD with at least a baseline visit and one follow-up visit 1 yr later were included in the study. In all study participants, the neuromuscular disease had been diagnosed $\geqslant 10$ yrs before study inclusion. None of the patients had performed the SNIP or PI,max manoeuvre prior to the study. Patients were included if they had been clinically stable for $\geqslant 1$ month. Noninvasive positive pressure ventilation was started when necessary according to international recommendations [9].

\section{Experimental protocol}

All pulmonary function tests were performed by the same experienced technicians in a single session for each patient at each visit. Patients were allowed time to recover fully between tests. Spirometry was performed in the sitting and supine positions. PI,max, SNIP and maximal expiratory pressure were recorded with the patient seated with PI,max and SNIP measured in random order.

\section{Spirometry}

At each visit, spirometric variables and lung volumes were measured using the Vmax 229 SensorMedics system (SensorMedics, Anaheim, CA, USA) according to standard guidelines [10]. The best of three reproducible values was recorded. Results were expressed as a percentage of published values [10].

\section{Respiratory muscle testing}

PI,max and SNIP were measured from functional residual capacity in a standard manner, as previously described elsewhere [4]. PI,max is an isometric manoeuvre and SNIP a quasi-isometric one $[2,5]$. PI,max was measured using a flanged mouthpiece with the manoeuvres repeated at least three times or until two identical readings were obtained [3]. Once the operator was satisfied, the maximum value of three manoeuvres that varied by $<20 \%$ was recorded [3]. SNIP was measured during $\geqslant 10$ and $\leqslant 20$ maximal sniffs in a standard manner, according to previously described methods [5]. Briefly, the plug used to obstruct the nostril was an eartip designed for recording auditory evoked potentials (Eartips, 13 mm; Nicolet Biomedical Inc., Madison, WI, USA). The plug was connected to a pressure transducer via a catheter, which was as short as possible. Air leakage was detected by obstructing the other nostril during an inspiratory manoeuvre and, when present, was eliminated by adding earplug wax. Detailed instructions as to how to perform the sniff manoeuvre were not given, as they were found to be unnecessary and possibly counterproductive in an earlier study [4]. Patients were encouraged vigorously during all test manoeuvres.

All pressure signals were measured using a differential pressure transducer (Validyne, Northridge, CA, USA), amplified by a carrier amplifier (Validyne) and passed through an analogue-digital board to a computer running AcqKnowledge software (Biopac Systems, Inc., Santa Barbara, CA, USA), which provided visual feedback to improve sniff efficiency. The signal was digitised at $100 \mathrm{~Hz}$. Patients received strong verbal encouragement in addition to the visual feedback, as suggested in a previous study [11]. The best values of $P_{\mathrm{I}, \mathrm{max}}$ and SNIP, expressed in $\mathrm{cmH}_{2} \mathrm{O}$, were recorded.

\section{Analysis}

Data from the DMD and MMD groups were compared using unpaired nonparametric Mann-Whitney U-tests. Changes in PI,max, SNIP and vital capacity (VC) in both populations were assessed using paired Wilcoxon tests.

The method, between SNIP and PI,max, that gave the highest value was considered the best method for the patient. Agreement between SNIP and PI,max was evaluated by constructing Bland-Altman plots [12]. Bias was represented by the mean differences between SNIP and PI,max. Upper and lower limits of agreement were defined as the 2.5 and $97.5 \%$ limits of the distribution of the differences. Precision (the ability to reproduce the same measurement) was represented by the interval (bias $\pm \mathrm{SD}$ ), where the $\mathrm{SD}$ was that of the distribution of differences.

Univariate and multivariate logistic regression analyses were performed in order to evaluate whether SNIP being better than PI,max was associated with the diagnosis (DMD or MMD) and/ or the severity of respiratory muscle weakness.

A p-value of $<0.05$ was considered significant.

\section{RESULTS}

The present study included 86 patients, 25 with DMD and 61 with MMD. All patients made two visits (representing $1 \mathrm{yr}$ of follow-up), 63 three visits (2 yrs of follow-up), 29 four visits (3 yrs of follow-up) and four five visits (4 yrs of follow-up).

\begin{tabular}{|c|c|c|}
\hline \multirow[t]{2}{*}{ TABLE 1} & \multicolumn{2}{|c|}{$\begin{array}{l}\text { Patient characteristics at baseline }(\mathrm{BL}) \text { and at the } \\
\text { second visit } 1 \mathrm{yr} \text { later }\end{array}$} \\
\hline & MMD & DMD \\
\hline Subjects n & 61 & 25 \\
\hline Age yrs $^{\#}$ & $42.2 \pm 11.6$ & $22.1 \pm 3.7$ \\
\hline Males/females & $42 / 19$ & $25 / 0$ \\
\hline BMI $\mathbf{k g} \cdot \mathbf{m}^{-2} \#$ & $25.8 \pm 6.2$ & $17.3 \pm 5.4$ \\
\hline \multicolumn{3}{|c|}{ Noninvasive mechanical ventilation $\mathbf{n}$} \\
\hline $\mathrm{BL}$ & 5 & 11 \\
\hline Visit 2 & 21 & 17 \\
\hline \multicolumn{3}{|l|}{ VC \% pred } \\
\hline $\mathrm{BL}$ & $63.4 \pm 16.9$ & $21.9 \pm 9.1$ \\
\hline Visit 2 & $62.0 \pm 18.0$ & $17.7 \pm 7.6^{*}$ \\
\hline \multicolumn{3}{|l|}{ SNIP $\mathrm{cmH}_{2} \mathrm{O}$} \\
\hline $\mathrm{BL}$ & $45.0 \pm 21.1$ & $20.2 \pm 9.5$ \\
\hline Visit 2 & $48.9 \pm 22.2$ & $16.3 \pm 9.0^{*}$ \\
\hline \multicolumn{3}{|l|}{$\mathrm{PI}, \max \mathrm{cmH}_{2} \mathrm{O}$} \\
\hline $\mathrm{BL}$ & $43.8 \pm 20.0$ & $24.2 \pm 9.7$ \\
\hline Visit 2 & $41.5 \pm 19.0$ & $21.3 \pm 7.6^{*}$ \\
\hline $\begin{array}{l}\text { Data are presen } \\
\text { muscular dystro } \\
\text { index; VC: vital } \\
\text { pressure; Pl,max: } \\
\text { versus BL (Wilco }\end{array}$ & $\begin{array}{l}\text { nted as mean } \pm S D \text {, unless otherwise stated. MN } \\
\text { ophy; DMD: Duchenne muscular dystrophy; BMI } \\
\text { I capacity; \% pred: \% predicted; SNIP: sniff nas } \\
\text { : maximal inspiratory pressure. }{ }^{\#} \text { : determined at } \\
\text { oxon test). }\end{array}$ & $\begin{array}{l}\text { : myotonic } \\
\text { body mass } \\
\text { inspiratory } \\
*: p<0.05\end{array}$ \\
\hline
\end{tabular}




\section{Baseline characteristics}

Table 1 reports the main patient characteristics at baseline; 16 patients used noninvasive mechanical ventilation. VC, SNIP and PI,max each differed significantly between DMD and MMD patients (Mann-Whitney test; $\mathrm{p}<0.0001$ ). A single DMD patient had a VC $>40 \%$ predicted, whereas only two MMD patients had VC $<40 \%$ pred.

Figures $1 \mathrm{a}$ and $\mathrm{b}$ show Bland-Altman plots of the difference between $P$ I,max and SNIP against the mean for each population. The bias (mean difference between SNIP and PI,max) in MMD patients at baseline was 1.2. The upper and lower limits of agreement were -29.5 and 32.0 , and the precision limits were -14.1 and 16.6. The bias for DMD patients at baseline was -4.0 . The upper and lower limits of agreement were -15.4 and 7.4, and the precision limits were -9.7 and 1.7. In summary, the limits of agreement were larger in MMD patients than in DMD patients. PI,max was better than SNIP in $20(80 \%)$ of the 25 DMD patients and $30(49 \%)$ of the 61 MMD patients.

The univariate logistic regression analyses performed to identify factors associated with better SNIP than PI,max at baseline showed correlations with VC and diagnosis (MMD or DMD) $(p=0.02$ and 0.004 , respectively). However, on multivariate logistic regression, only a diagnosis of MMD was significantly associated with SNIP being better than PI,max $(p=0.03)$.

\section{Changes over the first year}

Table 1 reports the main patient characteristics at the second visit. Mechanical ventilation was started during follow-up in 16 MMD and six DMD patients (table 1). VC, SNIP and PI,max each remained significantly different between MMD and DMD patients $(\mathrm{p}<0.0001$; Mann-Whitney test).

VC, SNIP and PI,max were significantly lower at the second visit in DMD patients $(\mathrm{p}<0.0015,0.03$ and 0.01 , respectively; Wilcoxon test) but not in MMD patients $(\mathrm{p}=0.09,0.06$ and 0.18 , respectively; table 2).

Figures 1c and d show Bland-Altman plots of the difference between PI,max and SNIP against the mean for each population. The bias for MMD patients at visit 2 was 7.4. The upper and lower limits of agreement were -23.9 and 38.8, and the precision limits were -8.2 and 23.1. The bias for DMD patients at visit 2 was -5.0 . The upper and lower limits of agreement were -20.0 and 10.0, and the precision limits were -12.5 and 2.5. SNIP was less than PI,max in $23(92 \%)$ of the 25 DMD patients and $19(31 \%)$ of the 61 MMD patients. In summary, the limits

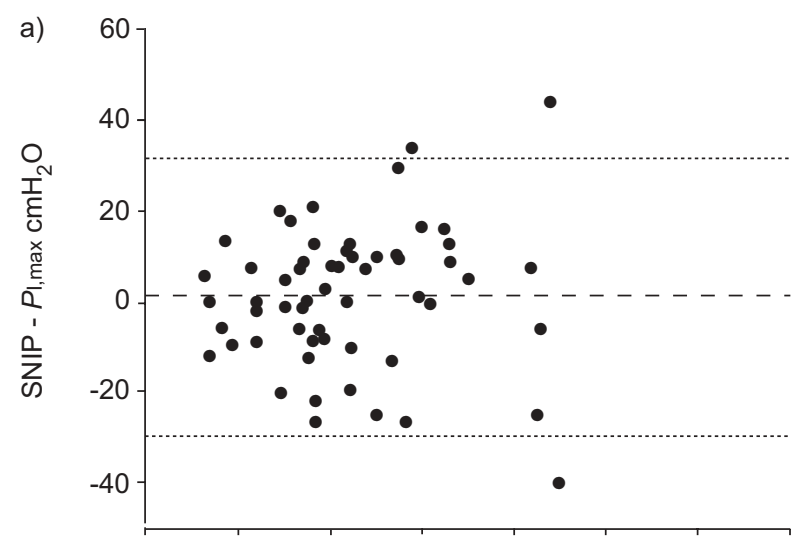

b)
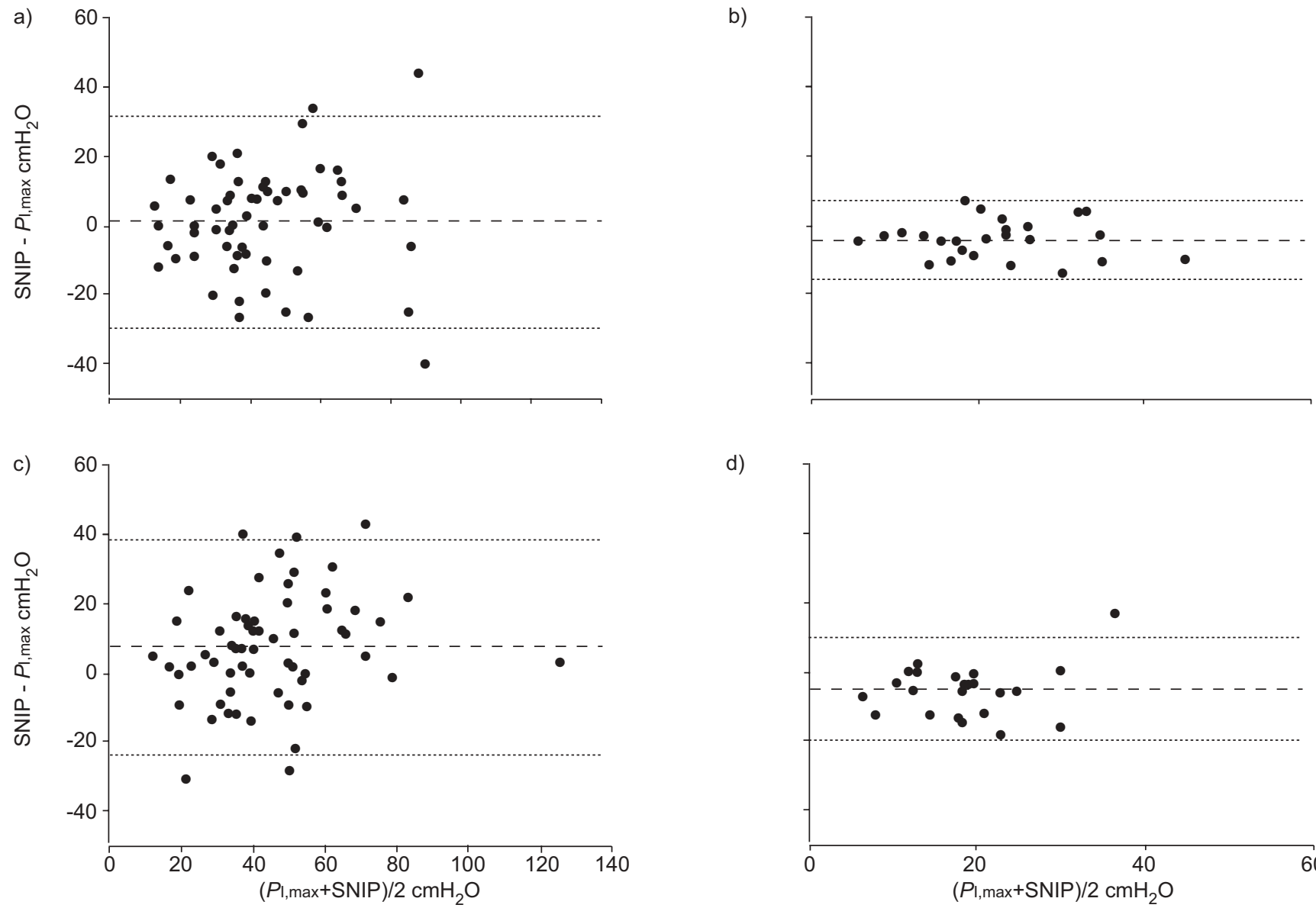

d)

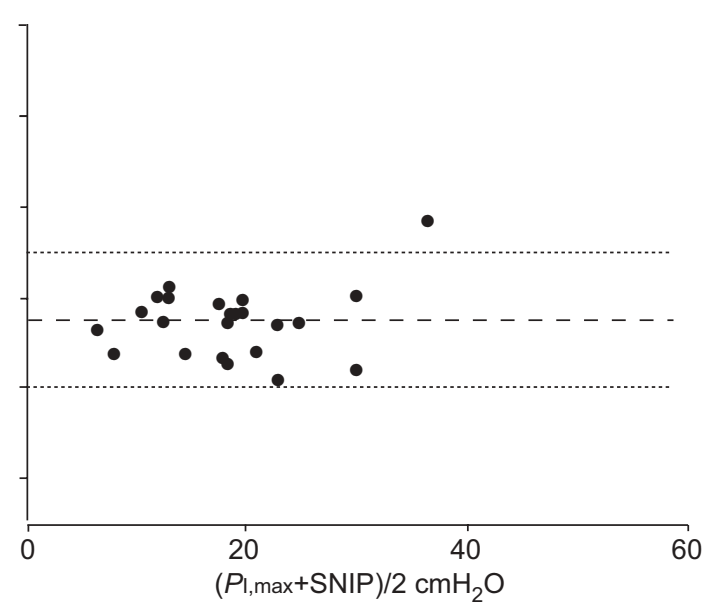

FIGURE 1. Bland-Altman plots showing the difference between sniff inspiratory nasal pressure (SNIP) and maximal static inspiratory pressure (PI,max) plotted against the mean of these two variables in a, c) Steinert myotonic muscular dystrophy patients $(n=61)$ and b, d) Duchenne muscular dystrophy patients $(n=25)$ at baseline $(a, b)$ and visit 2 (c, d). -----: mean; $\cdots \cdots \cdot \pm$ : 2 SD. 
TABLE 2 Baseline and visit 2 cross-tabulation

\begin{tabular}{|c|c|c|c|c|}
\hline & $P I$, max $-1>$ SNIP-1 & SNIP-1 $>$ PI,max-1 & $P I$, max $-1>$ SNIP-1 & SNIP-1 $>P_{1, \max -1}$ \\
\hline PI,max-2>SNIP-2 n & 20 & 3 & 15 & 4 \\
\hline SNIP-2 > PI,max-2 $\mathbf{n}$ & 0 & 2 & 15 & 27 \\
\hline
\end{tabular}

DMD: Duchenne muscular dystrophy; MMD: myotonic muscular dystrophy; PI,max-1: maximal inspiratory pressure (PI,max) at baseline; SNIP-1: sniff nasal inspiratory pressure (SNIP) at baseline; PI,max-2: PI,max at visit 2; SNIP-2: SNIP at visit 2

of agreement remained larger at visit 2 in MMD patients than in DMD patients. In addition, although PI,max remained better than SNIP in DMD patients (compared with baseline, the percentage of patients for whom PI,max was best increased from 80 to $88 \%$ of the DMD population), the number of MMD patient for whom $P_{\mathrm{I} \text {,max }}$ was better than SNIP decreased from $30(49 \%)$ to $19(31 \%)$.

In order to further evaluate changes in the best method ( $P$ I,max or SNIP) between the baseline visit and visit 2, a contingency table was built with two variables (baseline and visit 2; table 2). In the $20 \mathrm{DMD}$ patients with PI,max as the best method at baseline, $P$ I,max was still the best method after $1 \mathrm{yr}$. Of the five DMD patients with SNIP as the best method at baseline, three changed to PI,max being the best method after 1 yr. The best method changed between baseline and visit 2 in 15 of the 30 MMD patients with $P \mathrm{I}$,max as the best method at baseline and in four of the 31 MMD patients with SNIP as the best method at baseline.

\section{Long-term follow-up}

Since follow-up duration varied between patients, the best method (PI,max or SNIP) at baseline was crossed with the method that was most often best at subsequent visits (table 3 ). In the 17 DMD patients with PI,max as the best method at baseline, PI,max remained the best method throughout followup. In contrast, both DMD patients with SNIP as the best method at baseline switched to PI,max being the best method during follow-up. Of the $20 \mathrm{MMD}$ patients with PI,max as the best method at baseline, nine switched to SNIP during followup; of the 24 MMD patients with SNIP as the best method at baseline, only one switched to PI,max.

\section{DISCUSSION}

The present study comparing $P$ I,max and SNIP in patients with two of the most common chronic neuromuscular diseases showed that the test yielding the best value at baseline was usually $P \mathrm{I}$,max in DMD patients, whereas a more balanced distribution was seen in MMD patients. The best test changed significantly more often (30\%) in patients with MMD than in those with DMD (12\%). The frequency of switching from one test to the other differed according to which test yielded the best value at baseline. Thus, when PI,max was better than SNIP at baseline in DMD patients $(80 \%), P_{\mathrm{I}}$ max remained better $(100 \%)$ during follow-up. In contrast, when SNIP was better than $P \mathrm{I}, \mathrm{max}$ at baseline in DMD patients, a switch to $P \mathrm{I}, \max$ was often seen during follow-up. In MMD patients, when SNIP was better than PI,max at baseline (51\%), SNIP usually $(88 \%)$ remained better at the second visit; whereas when PI,max was better than SNIP at baseline, a switch was often (50\%) noted at the second visit.

The severity of respiratory function impairment differed between the two groups. However, it is well known that hypercapnia and a need for mechanical ventilation do not occur at the same level of restrictive disease in MMD patients as in DMD patients [13-16]. The need and reasons for monitoring respiratory function in the two populations in the present study were roughly similar, despite the difference in respiratory muscle function.

PI,max is the most widely used volitional test for evaluating inspiratory muscle strength [4]. However, many patients with neuromuscular disease find the PI,max manoeuvre difficult to perform. In addition, air leaks around the mouthpiece may be difficult to eliminate. The sniff test relies on a natural effort that

TABLE 3 Baseline and subsequent visit cross-tabulation

\begin{tabular}{|c|c|c|c|c|}
\hline & $P_{1, \text { max }}-1>$ SNIP-1 & SNIP-1 $>P I$, max-1 & PI,max-1>SNIP-1 & SNIP-1 $>$ PI,max-1 \\
\hline$P$ I,max-FU $>$ SNIP-FU & 17 & 2 & 11 & 1 \\
\hline SNIP-FU $>P I$, max-FU & 0 & 0 & 9 & 23 \\
\hline
\end{tabular}


most patients find easier to perform than the $P_{\mathrm{I}, \max }$ manoeuvre [17]. SNIP is a more pleasant technique than PI,max for most patients $[6,18,19]$ and it does not raise the leak problems with a mouthpiece sometimes observed in neuromuscular patients. SNIP may carry less risk of fatigue, as the manoeuvre is shorter than $P \mathrm{I}$,max, which requires a sustained peak pressure for $\geqslant 1 \mathrm{~s}$. Predicted normal values adjusted for age and sex are available in the literature for both adults and children [5, 19-21]. Based on these normative values, it is generally considered that a SNIP of $<-70 \mathrm{cmH}_{2} \mathrm{O}$ in males or $<-60 \mathrm{cmH}_{2} \mathrm{O}$ in females is unlikely to be associated with inspiratory muscle weakness [4, 20, 22].

SNIP reflects the oesophageal pressure decrease during the sniff manoeuvre, which accurately reflects inspiratory muscle strength in normal individuals [5]. However, SNIP may underestimate inspiratory muscle strength in specific populations, such as individuals with nasal obstruction [21] and patients with chronic obstructive pulmonary disease (since sniff is a short manoeuvre and transmission of oesophageal pressure to the nose is reduced in these patients [23]). Underestimation probably occurred in patients with severe neuromuscular disease who were unable to produce an initial negative transnasal pressure of $10-15 \mathrm{cmH}_{2} \mathrm{O}$, a necessary condition for collapsing the unplugged nostril valve [24]. In order to obtain this negative transnasal pressure, the patient must produce an oesophageal pressure swing of $>10$ $15 \mathrm{cmH}_{2} \mathrm{O}$, which depends on inspiratory peak flow and on nostril resistance when the valve is not collapsed. In addition, SNIP is generated in a ballistic manoeuvre during which the inspiratory muscles shorten to a greater extent and at a higher speed than during the PI,max manoeuvre, which is a more sustained isometric effort. Given both the force-velocity and force-length relationships of striated muscle, SNIP should be $<P$ I,max, as pressure generation falls when the operating length of the muscle decreases and when the velocity of muscle shortening increases. Accordingly, PI,max was $>$ SNIP in patients with severe neuromuscular disease [7, 25]. The present results are in agreement with these previous studies; muscle weakness was more severe in the DMD group than in the MMD group, and PI,max yielded the best value at baseline in most DMD patients, whereas a more balanced distribution was noted in the MMD patients.

Sniffing is more natural than the PI,max manoeuvre, a fact believed to explain the higher SNIP than PI,max in normal individuals [6] and patients with limited respiratory muscle weakness $[7,25]$, such as those in the present MMD group. In addition, the SNIP is measured from the peak pressure, whereas PI,max is the mean pressure sustained over $1 \mathrm{~s}$, which includes an early pressure peak followed by a decline to a lower sustained pressure. Thus, the nature of the predominant effect influences the SNIP relative to the PI,max. Finally, due to the differences in the type of effort and pattern of muscle activation between the two manoeuvres, SNIP and PI,max probably reflect different aspects of inspiratory muscle function. A more sustained manoeuvre may achieve greater inspiratory muscle activation in patients with more severe weakness.

In addition to the severity of respiratory muscle weakness, a number of other factors may contribute to the difference between the DMD and MMD groups regarding the method producing the best value. Logistic regression analysis was performed to look for factors associated with a better SNIP than PI,max at baseline. When VC and diagnosis were entered in a univariate analysis, correlations were observed with both factors ( $p=0.02$ and 0.004 , respectively). However, when these factors were combined in an multivariate logistic regression analysis, only diagnosis was significantly associated with SNIP being better than $P_{I}, \max (\mathrm{p}=0.03)$. PI,max and SNIP are both volitional methods that require cooperation and motivation from the patient. The greater variability in the patients with MMD may be ascribable to lack of motivation and poor coordination. MMD is an autosomal dominant multisystemic disorder that often involves the brain and results in cognitive impairment [26], thereby possibly increasing the variability in volitional manoeuvres. Interestingly, the considerable variability in the MMD group regarding the best test was observed only when PI,max was better at baseline, suggesting that the ability to perform SNIP properly persisted over time. Conversely, some of the MMD patients who were unable to perform SNIP properly at baseline learnt the manoeuvre during follow-up. The ability to perform PI,max, a more difficult and less natural manoeuvre than SNIP, was lost over time in some patients. Cognitive tests were not performed, and thus it was not possible to assess possible relationships between variability in the best test and cognitive impairment.

In order to assess the hypothesis that a better SNIP than PI,max is more likely to occur in patients with mild rather than severe muscle weakness, NICOT et al. [27], a group from a paediatric pulmonary department with extensive experience in respiratory muscle function testing in neuromuscular patients, were asked to compare SNIP and PI,max in 15 DMD children with VC $>40 \%$ pred. SNIP was better than PI,max in eight patients, a proportion similar to that seen in the current MMD population. This finding supports the present hypothesis.

In conclusion, these two volitional tests are not interchangeable in patients with chronic neuromuscular disorders [6-8]. Nevertheless, the use of sniff nasal inspiratory pressure measurement has only been suggested for monitoring patients with amyotrophic lateral sclerosis $[18,28]$. In the present adult patients with Duchenne muscular dystrophy, the maximal inspiratory pressure manoeuvre generally yielded the best value at baseline, and maximal inspiratory pressure assessment remained the best test in most patients during follow-up. Thus, maximal inspiratory pressure measurement alone may be sufficient in this adult population. In patients with myotonic muscular dystrophy, in contrast, the marked variability in the test yielding the best value at baseline indicates a need for performing both tests at baseline. During follow-up, when sniff nasal inspiratory pressure measurement yielded the best value at baseline, sniff nasal inspiratory pressure remained best during follow-up. Therefore, using sniff nasal inspiratory pressure measurement alone during follow-up may be appropriate in this subpopulation provided vital capacity remains $>40 \%$ pred. Nevertheless, maximal inspiratory pressure and sniff nasal inspiratory pressure manoeuvres should both be performed when the patient shows poor cooperation or difficulty in understanding and/or performing the manoeuvre. 


\section{REFERENCES}

1 Black LF, Hyatt RE. Maximal static respiratory pressures in generalized neuromuscular disease. Am Rev Respir Dis 1971; 103: 641-650.

2 Black LF, Hyatt RE. Maximal respiratory pressures: normal values and relationship to age and sex. Am Rev Respir Dis 1969; 99: 696-702.

3 Wilson SH, Cooke NT, Edwards RH, Spiro SG. Predicted normal values for maximal respiratory pressures in Caucasian adults and children. Thorax 1984; 39: 535-538.

4 American Thoracic Society, European Respiratory Society, ATS/ERS statement on respiratory muscle testing. Am J Respir Crit Care Med 2002; 166: 518-624.

5 Héritier F, Rahm F, Pasche P, Fitting JW. Sniff nasal pressure. A noninvasive assessment of inspiratory muscle strength. Am J Respir Crit Care Med 1994; 150: 1678-1683.

6 Stefanutti D, Benoist MR, Scheinmann P, Chaussain M, Fitting JW. Usefulness of sniff nasal pressure in patients with neuromuscular or skeletal disorders. Am J Respir Crit Care Med 2000; 162: 1507-1511.

7 Hart N, Polkey MI, Sharshar T, et al. Limitations of sniff nasal pressure in patients with severe neuromuscular weakness. J Neurol Neurosurg Psychiatry 2003; 74: 16851687.

8 Prigent H, Lejaille M, Falaize L, et al. Assessing inspiratory muscle strength by sniff nasal inspiratory pressure. Neurocrit Care 2004; 1: 475-478.

9 Clinical indications for noninvasive positive pressure ventilation in chronic respiratory failure due to restrictive lung disease, COPD, and nocturnal hypoventilation - a consensus conference report. Chest 1999; 116: 521-534.

10 Quanjer PH, Tammeling GJ, Cotes JE, Pedersen OF, Peslin R, Yernault JC. Lung volumes and forced ventilatory flows. Report Working Party Standardization of Lung Function Tests, European Community for Steel and Coal. Official Statement of the European Respiratory Society. Eur Respir J 1993; 6: Suppl. 16, 5-40.

11 Laporta D, Grassino A. Assessment of transdiaphragmatic pressure in humans. J Appl Physiol 1985; 58: 1469-1476.

12 Bland JM, Altman DG. Statistical methods for assessing between two methods of clinical measurement. Lancet 1986; 1: 307-310.

13 Nugent AM, Smith IE, Shneerson JM. Domiciliary-assisted ventilation in patients with myotonic dystrophy. Chest 2002; 121: 459-464.

14 Hukins CA, Hillman DR. Daytime predictors of sleep hypoventilation in Duchenne muscular dystrophy. Am J Respir Crit Care Med 2000; 161: 166-170.
15 Nickol AH, Hart N, Hopkinson NS, Moxham J, Simonds A, Polkey MI. Mechanisms of improvement of respiratory failure in patients with restrictive thoracic disease treated with non-invasive ventilation. Thorax 2005; 60: 754-760.

16 Bégin P, Mathieu J, Almirall J, Grassino A. Relationship between chronic hypercapnia and inspiratory-muscle weakness in myotonic dystrophy. Am J Respir Crit Care Med 1997; 156: 133-139.

17 Laroche CM, Mier AK, Moxham J, Green M. The value of sniff esophageal pressures in the assessment of global inspiratory muscle strength. Am Rev Respir Dis 1988; 138: 598-603.

18 Fitting JW, Paillex R, Hirt L, Aebischer P, Schluep M. Sniff nasal pressure: a sensitive respiratory test to assess progression of amyotrophic lateral sclerosis. Ann Neurol 1999; 46: 887-893.

19 Stefanutti D, Fitting JW. Sniff nasal inspiratory pressure. Reference values in Caucasian children. Am J Respir Crit Care Med 1999; 159: 107-111.

20 Uldry C, Fitting JW. Maximal values of sniff nasal inspiratory pressure in healthy subjects. Thorax 1995; 50: 371-375.

21 Fitting JW, Heritier F, Uldry C. Evaluation de la force musculaire inspiratoire par la pression nasale lors du sniff. [Evaluation of the inspiratory muscle strength using the nasal pressure of the sniff.] Rev Mal Respir 1996; 13: 479-484.

22 Polkey MI, Green M, Moxham J. Measurement of respiratory muscle strength. Thorax 1995; 50: 1131-1135.

23 Uldry C, Janssens JP, de Muralt B, Fitting JW. Sniff nasal inspiratory pressure in patients with chronic obstructive pulmonary disease. Eur Respir J 1997; 10: 1292-1296.

24 Haight JS, Cole P. The site and function of the nasal valve. Laryngoscope 1983; 93: 49-55.

25 Iandelli I, Gorini M, Misuri G, et al. Assessing inspiratory muscle strength in patients with neurologic and neuromuscular diseases: comparative evaluation of two noninvasive techniques. Chest 2001; 119: 1108-1113.

26 Meola G, Sansone V, Perani D, et al. Executive dysfunction and avoidant personality trait in myotonic dystrophy type 1 (DM-1) and in proximal myotonic myopathy (PROMM/ DM-2). Neuromuscul Disord 2003; 13: 813-821.

27 Nicot F, Hart N, Forin V, et al. Respiratory muscle testing: a valuable tool for children with neuromuscular disorders. Am J Respir Crit Care Med 2006; 174: 67-74.

28 Lyall RA, Donaldson N, Polkey MI, Leigh PN, Moxham J. Respiratory muscle strength and ventilatory failure in amyotrophic lateral sclerosis. Brain 2001; 124: 2000-2013. 